Ann. Abeille, 1963, 6 (3), 235-244.

\title{
VARIATIONS DES ASSOCIATIONS POLLINIQUES OBSERVÉES DANS DES MIELS RÉCOLTÉS EN UN MÊME LIEU PAR DES ABEILLES APPARTENANT A DES SOUS-ESPÈCES DIFFÉRENTES
}

\author{
J. LOUIS
}

Station de Recherches sur l'Abeille et les Insectes sociaux, Bures-sur-Yvette (Seine-et-Oise)

\section{SOMMAIRE}

L'auteur étudie les fluctuations des associations polliniques mises en évidence dans des miels récoltés dans la région du Jura par des colonies d'A. $m$. mellifica, $A$. m. caucasica, $A$. $m$. ligustica et $A$. $m$. carnica réunies en un même rucher et soumises aux mêmes conditions de butinage. L'analyse des résultats montre un rapport entre la longueur du proboscis et l'aptitude des ouvrières à butiner sur le Trèfle violet ( $T r$. pratense), sur lequel certaines sous-espèces rencontrent des difficultés qu'elles ne peuvent parfois surmonter pour atteindre les nectaires.

\section{I. - INTRODUCTION}

La présente étude a été réalisée à la demande des apiculteurs récoltants qui souhaitent connaître avec plus de précisions le comportement des abeilles d'origine locale et des sous-espèces couramment importées en France, envers les différentes associations florales spontanées ou artificielles qui constituent le tapis végétal français. La "Compagnie des miels du Jura (1) " a fourni le matériel nécessaire à cette étude. Le travail a donc été effectué sur des échantillons provenant du département du Jura. Il est probable que les résultats acquis sont également valables en d'autres lieux. Il serait toutefois souhaitable d'en apporter ultérieurement la confirmation.

(1) L'auteur remercie les membres de la Compagnie des miels du Jura et particulierement II. BorNECK qui a eu une action décisive dans l'initiative et la préparation de ce travail. 
En I95I, GoETzE a effectué une étude assez approfondie des relations qui existent entre la longueur de la langue de l'abeille allemande et la récolte du nectar sur Tr. pratense. Plus récemment, A. Maurizio (I962) a publié un travail portant surtout sur l'analyse pollinique et les différences que cette technique peut mettre en évidence quant à la composition florale des miels récoltés par des abeilles de provenances distinctes. Les effets de la longueur exceptionnelle du proboscis de $A . m$. caucasica n'ont pas encore été précisés en France.

\section{MATÉRIEI, ETT MÉTHODE}

Io) MÁTÉRIEL,

a) Les ruches

Les Reines caucasiennes ont été importées d'U. R. S. S. et installées en France en septembre r 960.

Les abeilles noires proviennent d'essaims naturels et d'un élevage de reines effectué au cours de la saison I 960 aux Arsures près d'Arbois (Jura).

Les Reines carnioliennes ont été importées et installées en Août 1960. Elles étaient originaires de Yougoslavie.

Des hybrides italiennes existaient déjà très probablement dans le rucher ou à proximité et dérivaient de souches pures importées antérieurement.

\section{b) Les échantillons}

Les abeilles ont été prélevées directement sur les cadres et expédiées dans des manchons de toile métallique. Chaque échantillon comptait environ une centaine d'individus. Les prélèvements ont été effectués au cours de la saison qui a suivi l'installation des ruches dans le rucher expérimental ( 1961$)$. Dès leur arrivée au laboratoire, les abeilles ont été tuées par le froid et conservées ainsi à basse température jusqu'à examen.

\section{0) MÉTHODES}

Les observations et les mesures ont été effectuées à la loupe binoculaire (objectif $\times \mathrm{I}$, oculaire $\times 25$ ), sur des échantillons uniformément composés de zo abeilles par ruche. Nous a vons basé nos diagnostics de subspéciation sur l'examen de cinq̨ caractères définis antérieurement par GoETZE 1960). Nous en rappelons ci-dessous l'essentiel pour une meilleure compréhension du tableau 2.

La longueur de la langue a fait l'objet d'une étude séparée. Afin que les mesures soient comparables d'un individu à l'autre, la glosse a été mise avec soin en extension maximum, mais toutefois, sans étirement ni contrainte. Les mesures sont exprimées en millimètres et dixièmes de millimètres.

\section{$3^{\circ}$ ) DESCRIPTIONS DES CARACTÈrES MORPHOLOGIQUES}

Les quatre premiers caractères ont été estimés et répartis en trois catégories, l'une moyennę et deux extrêmes. Le second (Toison) a fait l'objet de mensurations complémentaires. Les caractères "index cubital " et "langue " ont été mesurés.

\section{a) Couleur}

Ce caractère est apprécié sur le premier tergite abdominal (après le propodeum). On a distingué :

- les abeilles noires, c'est-à-dire celles qui présentent une coloration noire quasi totale de tous les segments (tableau 2 ; col. 3 ). 


\begin{tabular}{|c|c|c|}
\hline \multicolumn{2}{|c|}{$\dot{0} \dot{g}$} & $\dddot{2} 25=29$ \\
\hline \multicolumn{2}{|c|}{ 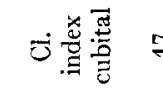 } & 2925 \\
\hline \multicolumn{2}{|c|}{ 希焉 } & 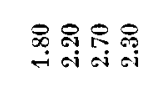 \\
\hline \multirow{3}{*}{ 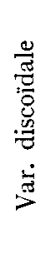 } & + & - 屏屏 \\
\hline & $0=$ & $\stackrel{20}{\rightarrow}$ \\
\hline & $1=$ & 29 \\
\hline \multirow{3}{*}{ 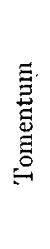 } & $\sim q$ & ๑๑尺 \\
\hline & $z=$ & $B$ \\
\hline & $x \leqslant$ & \\
\hline \multicolumn{2}{|c|}{ 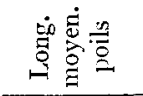 } & 1= ו: 0: \\
\hline \multirow{3}{*}{ 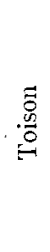 } & $-i$ & \\
\hline & $\Rightarrow$ & 9 \\
\hline & 0 & 용 \\
\hline \multirow{3}{*}{$\frac{\bar{a}}{3}$} & $\mapsto \quad$ & 8 \\
\hline & $\rightleftarrows$ & \\
\hline & z & 토용 \\
\hline \multicolumn{2}{|c|}{ 吕悹总 } & $e 00$ \\
\hline \multicolumn{2}{|c|}{ 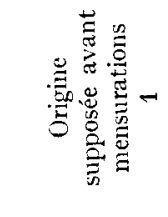 } & 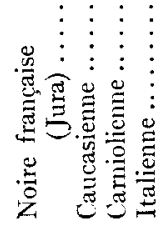 \\
\hline
\end{tabular}

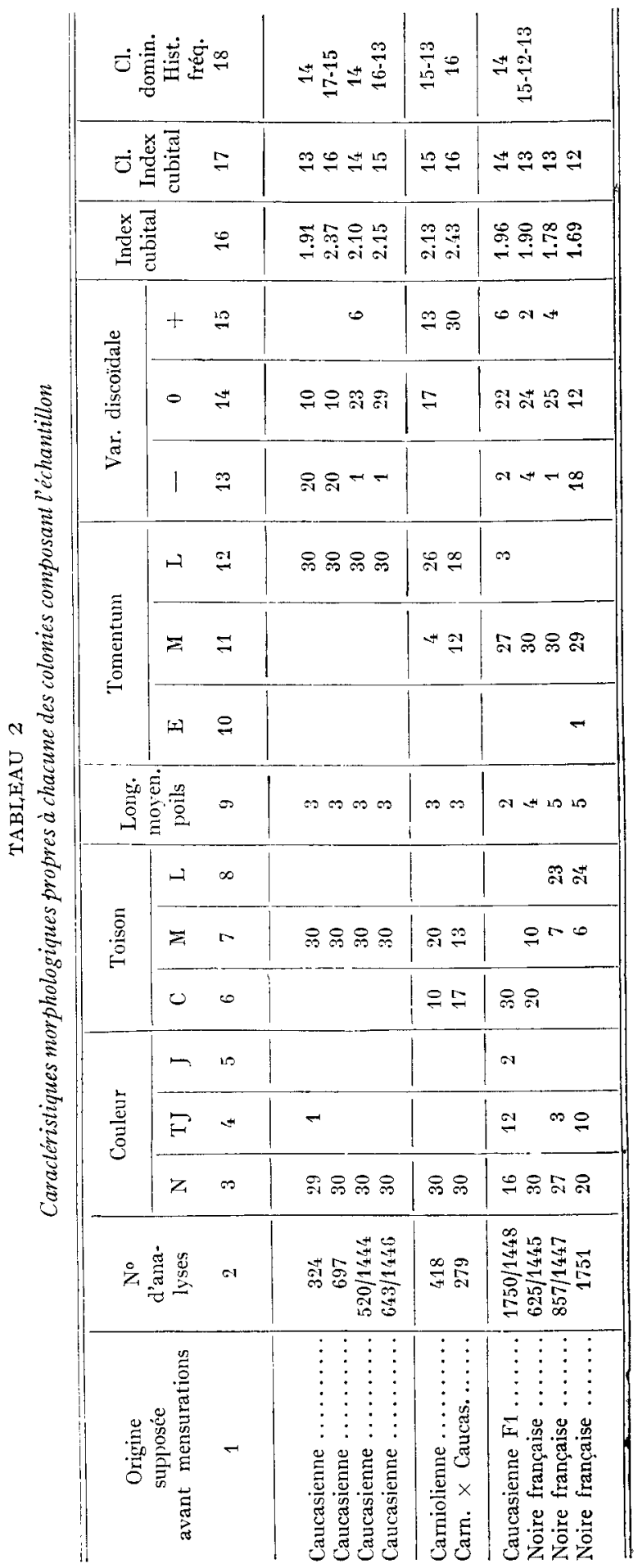


- Les abeilles à taches jaunes qui ont deux taches symétriques d'étendue limitée de part et d'autre à la partie supérieure du même segment (tableau 2 ; col. 4).

- Les abeilles jaunes qui présentent, elles, une décoloration totale du premier segment, s'étendant même sur le second et les suivants (tableau 2 ; col. 5 ).

\section{b) Longueur de la toison}

On exprime le caractère en observant, de profil, la longueur de la pilosité qui se dresse sur l'avant-dernier tergite abdominal.

- courte $\quad 2$ à 3 dixièmes de millimètre en moyenne

- moyenne $\quad 3$ à 4 dixièmes de millimètre en moyenne

- longue $\quad 4$ à 5 dixièmes de millimètre en moyenne

Les colonnes 6,7 et 8 du tableau 2 précisent le nombre d'abeilles appartenant à chacune de ces catégories, la colonne 9, la longueur moyenne de la toison en $1 /$ Io de $\mathrm{mm}$ calculée sur $3^{\circ}$ abeilles.

\section{c) Largeur des bandes du tomentum}

On observe cette caractéristique sur le pénultième ou l'antépénultième tergite. On apprécie ou l'on mesure la partie recouverte de poils par rapport à la partie non ouverte. Étant donné les différences importantes qui existent entre les abeilles françaises, caucasiennes et carnioliennes, nous n'avons pas cru devoir exprimer un index du tomentum. C'est généralement dans ce cas un rapport numérique établi entre les mesures de la partie pileuse et celles de la partie glabre.

- bandes étroites

- bandes moyennes tableau 2 ; col. Io

- bandes larges tableau 2 ; col. I I tableau 2 ; col. 12

\section{d) Variation du point discoïdal}

Ce caractère est désigné par GoEtze par le terme "Discoidalverschiebung ». Le point discoïdal est le sommet de l'angle apical inférieur de la deuxième cellule discoïdale de l'aile. On observe la variation de la position de ce point par rapport à une droite obtenue de la façon suivante : sous la loupe et à l'aide d'un réticule oculaire, on joint les sommets de la cellule radiale ; puis par le point de convergence de la troisième transverso-cubitale avec la nervure inférieure de la cellule radiale, on trace la perpendiculaire au grand axe de la cellule radiale qui en joint les sommets. On observe enfin la position du point discoïdal par rapport à cette perpendiculaire.

- Si le point est situé du côté de l'attache de l'aile, la position est dite négative (-) tableau 2

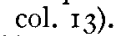

- Si le point est situé sur la perpendiculaire, la position est dite nulle (o) (tableau 2, col. I4),

- Si le point est situé du côté de l'axe de l'aile, la position est dite positive $(+)$ (tableau 2. col. I 5 ).

\section{e) Index cubital}

L'index cubital est un rapport établi entre les deux portions de nervures qui constituent la partie inférieure de la troisième cellule cubitale (tableau 2, col. I6). Les rapports ont été regroupés selon les classes calculées par Dreher (1950), dans le but de rétablir la normalité de la distribution. La classe de l'index cubital est exprimée dans la colonne I 7 . L'histogramme est sommairement décrit dans la colonne 18 . (L'indication de deux classes, ou plus, signifie une distribution bi ou tri-modale probable).

\section{$\left.4^{\circ}\right)$ CHIFHRES THÉORIQUES DE RÉFÉRENCES}

Des chiffres théoriques de références ont été établis, d'une part à partir de données recueillies dans la bibliographie, d'autre part à partir de données personnelles non publiées. Ils ont été réunis dans le tableau $\mathbf{r}$ auquel on pourra se reporter pour effectuer des comparaisons avec le tableau 2. 


\section{RÉSULTATS}

\section{A. - Exposé ET INTERPRÉtTation dEs RÉSUltats DE L'ANALYSE MORPHOLOGIQUE QUANTITATIVE}

I ${ }^{\circ}$ Description quantitative des caractéristiques morphologiques générales des colonies composant l'échantillon

Le tableau $\mathrm{No}_{2}$ contient les éléments de description numérique.

$\left.2^{\circ}\right)$ Diagnostic de subspéciation des colonies

\section{a) Aboilles caucasiennes.}

Colonie $n^{\circ} 324$. - Cette colonie possède de façon stable les caractéristiques de l'abeille caucasienne. Uniformément noire, elle présente, dans un cas seulement, une très légère décoloration. Les poils de la toison sont moyens, de l'ordre de $3 /$ Io de $\mathrm{mm}$, les bandes du tomentum sont très larges donnant à l'abeille un aspect gris typique. L'index cubital moyen est peut-être un peu faible, cependant la plus grande fréquence des mesures se situe dans la classe I4, c'est-à-dire au-dessus de 2,00.

Colonie no 697. - Cette colonie montre les mêmes caractéristiques que la précédente à cette exception près que l'histogramme de fréquence de l'index cubital présente deux sommets. Pourtant il s'agit là vraisemblablement d'abeille caucasienne non hybridée. On sait que celle-ci est en son lieu d'origine très variable. Il semble que nous soyons en présence de deux variantes à l'intérieur d'une même colonie d'abeilles caucasiennes.

Colonie $n^{0}$ 520. - Mêmes caractéristiques que les précédentes.

Colonie $n^{\circ} 643$. - Cette colonie est à forte dominance caucasienne. Toutefois, la distribution des fréquences des mesures de l'index cubital est bimodale. On remarque la présence d'abeilles montrant un index cubital correspondant à l'Abeille noire française (classe I3, I,7, I, 8). Nous pensons qu'un doute est possible quant à sa pureté, bien que la variation soit normale et que ce doute ne puisse être confirmé par un autre caractère.

\section{b) Abeilles hybrides.}

Colonies $\mathrm{n}^{\mathrm{os}} 418$ et 279 . - Ces deux ruches proviennent d'un croisement entre les variétés carniolienne et caucasienne. La mise en évidence de sang carniolien est caractérisée notamment par la présence de poils courts et un fort pourcentage de translations positives du point discoïdal.

Colonie $n^{0} 1750$. - Lors d'un rapide examen sur le terrain, celle-ci a été tout d'abord considérée comme une ruche caucasienne. L'analyse approfondie au laboratoire a permi de déceler une fécondation possible par un ou plusieurs mâles italiens. La présence, d'une part, de près de $50 \mathrm{p}$. Ioo d'abeilles présentant des taches jaunes et même entièrement jaunes, d'autre part la présence poils très courts, en apportent la preuve certaine. Enfin, les bandes du tomentum sont également moins larges que chez l'Abeille caucasienne typique.

Colonie $n^{0} 625$. - De même que pour la colonie précédente, un examen sommaire nous avait porté à conclure qu'il s'agissait d'abeilles noires d'origine locale. 
Or, il s'agit en réalité, de caucasiennes fortement hybridées de noires françaises avec des traces d'abeilles carnioliennes. L'histogramme de fréquence des index cubitaux présente trois sommets. La variation est élevée. On remarque également un fort pourcentage de poils courts (67 p. IOO).

c) A beilles noires trançaises.

Colonies $n^{\circ} 857$ et 1751 . - Ces deux ruches correspondent exactement aux caractéristiques de 1'abeille noire française du Jura avec un index cubital oscillant entre les classes 12 et $I_{3}(\mathrm{r}, 63-\mathrm{I}, 78$ et $\mathrm{I}, 78-\mathrm{I}, 94)$.

$\left.3^{\circ}\right)$ Variation entre sous-espèces de la longueur du proboscis

TABIEAU 3

Étude comparative de la longueur de la langue

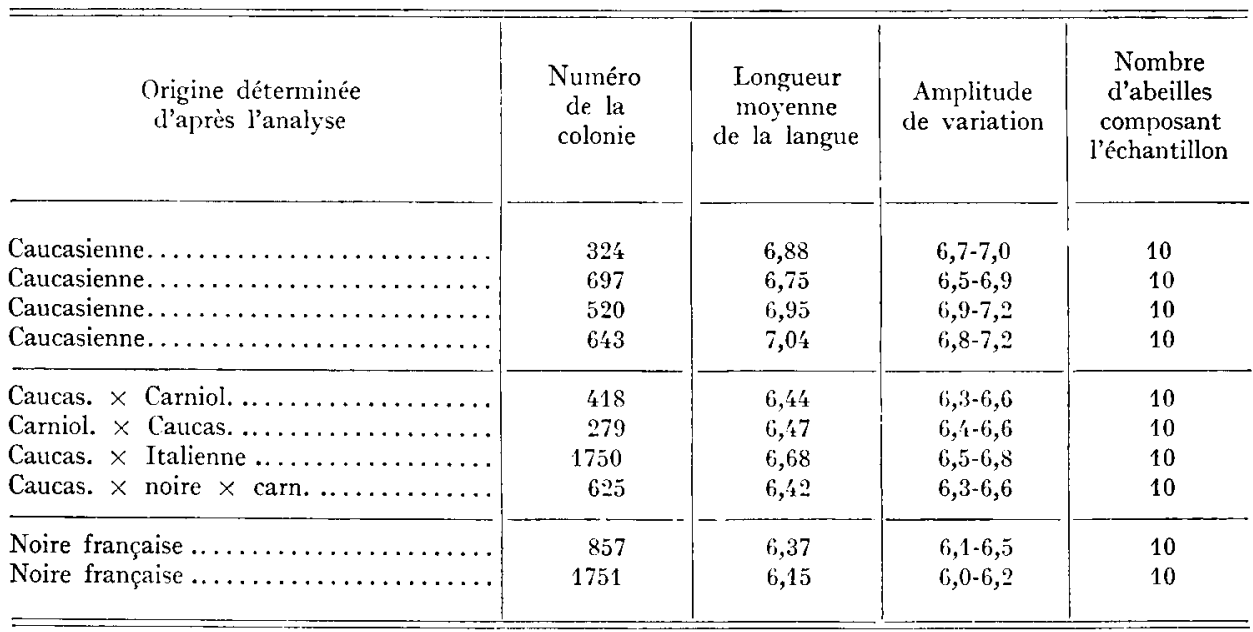

On remarque qu'en ce qui concerne le groupe de type catucasien, (colonies nos $324,697,520,643$ ) la longueur de la langue est voisine de $7 \mathrm{~mm}$ alors que les colonies hybrides ( $\left.\mathrm{n}^{\mathrm{Os}} 4 \mathrm{I} 8,279,175^{\circ}, 625\right)$ se situent aux environs de 6,5. Enfin, les colonies noires (nos 857 et I 75I) ont une langue très courte par rapport aux deux autres sousespèces. La taille du proboscis est plutôt voisine de $6 \mathrm{~mm}$. Pour l'ensemble de l'échantillonnage, il apparaît que la mesure la plus basse se situe à $6 \mathrm{~mm}$ et la plus élevée à $7,2 \mathrm{~mm}$, soit $\mathrm{r}, 2 \mathrm{~mm}$ d'écart entre la forme caucasienne et la forme française. Cette différence représente environ $\mathrm{r} / 2 \mathrm{O}^{\mathrm{e}}$ de la longueur du corps de l'ouvrière. La différence entre les valeurs moyennes n'est que de $0,7 \mathrm{~mm}$, mais du point de vue pratique et en ce qui concerne la récolte du nectar, la présence et le nombre des individus ayant une langue très longue est au moins aussi important que les valeurs moyennes moins descriptives. Nous remarquons également qu'il n'y a pas de chevauchement entre les valeurs extrêmes relevées chez les Abeilles françaises comparées à celles des Abeilles caucasiennes. Ce fait rend tout test statistique superflu, la différence étant d'emblée hautement significative entre $A . m$. mellifica et $A . m$. caucasica. 


\section{B. 一 EXPOSÉ ET INTERPRÉTATION DES RÉSULTATS DE I'ANALYSE POLLINIQUE ( ${ }^{1}$ )}

\section{I) Renseignements généraux}

L,e rucher expérimental était situé sur le territoire de la commune de Villeneuve d'Aval (Jura), à une altitude de 250 mètres. Le sol est composé d'argiles marneuses et d'alluvions siliceuses. La flore comprend des bois de chênes et de hêtres. Ces bois sont bordés de prairies naturelles pauvres. Le trèfle violet (Tr. pratense) se trouve dans quelques prairies artificielles. On remarque également du trèfle blanc dans les pâtures.

\section{TABLEAU 4}

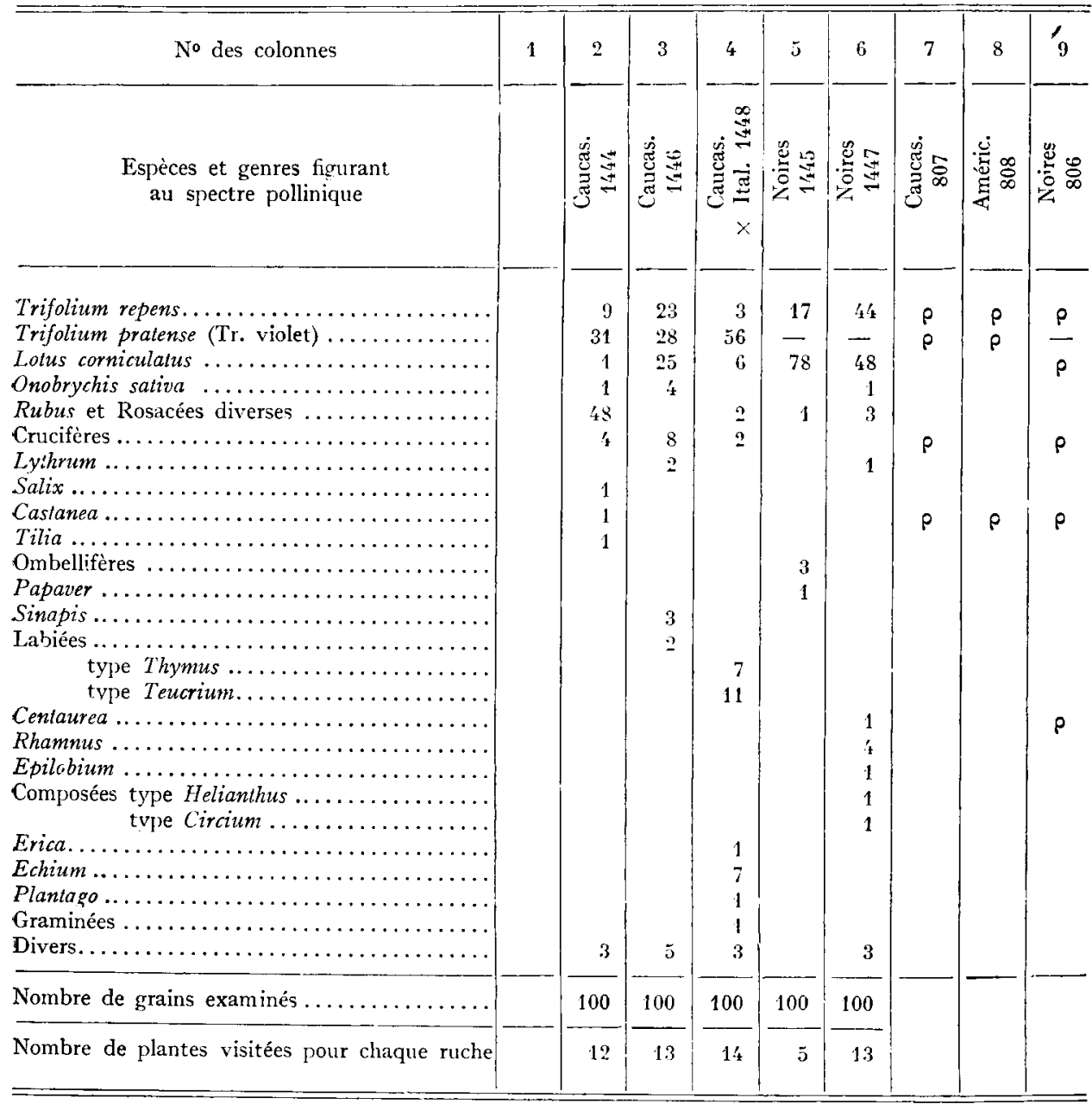

(1) L'auteur remercie M. LovveAux d'avoir bien voulu accepter de lui apporter son aide précieuse et de garantir ainsi toute la partie relative à la melisso-palynologie. 
Les échantillons de miel ont été prélevés directement dans les rayons avant la récolte, et n'ont subi qu'un filtrage grossier. D'après 1'aspect et la couleur des opercules, les échantillons correspondaient parfaitement à la totalité du miel qui était alors en hausse.

Les analyses polliniques ont été effectuées selon les techniques décrites en 1952 par A. Maurizio et Louveaux, lors de la réunion de Commision internationale de Botanique apicole.

\section{$\left.2^{\circ}\right)$ Résultats des analyses et discussion}

Les colonnes 2 à 6 correspondent à cinq analyses qualitatives et quantitatives effectuées chacune sur Ioo grains de pollen; les trois dernières colonnes $(7,8,9)$ ne correspondent qu’à trois analyses qualitatives effectuées antérieurement, et qui confirment nos observations. Le tableau no 4 met en évidence :

I - qu'aucune des ruches de race française n'a travaillé sur Trèfle violet ( $T r$. pratense) (col. 5 et 6 confirmées par la colonne 9). Les récoltes ont été effectuées de préférence sur trèfle blanc et lotier ;

2 - que les deux ruches caucasiennes ont travaillé sur Tr. pratense dans une proportion voisine de $30 \mathrm{p}$. Ioo ;

3 - qu'il faut tenir compte également que, bien que la moyenne de la colonie hybride (col. $n^{\circ} 4$ ) se situe, en ce qui concerne la longueur de la langue, entre l'Abeille noire et l'Abeille caucasienne, celle-ci semble avoir travaillé sur Trèfle violet dans une proportion presque double de celle des caucasiennes.

Ce dernier résultat ne peut cependant être considéré que comme une indication car il ne repose que sur une seule observation qui devra être infirmée ou confirmée ultérieurement.

Les autres pourcentages ne figurent qu'à titre indicatif car ils n'apportent pas d'informations complémentaires. A l'exception du Trèfle violet, on ne peut donc pas dire que, dans les conditions de l'expérience, les Abeilles caucasiennes s'intéressent à des espèces végétales différentes de celles qui sont butinées par 1'Abeille française.

\section{TABLEAU 5}

La coloration est en progression inverse du pourcentage de grains de pollen de Trifolium pratense

\begin{tabular}{|c|c|c|c|c|}
\hline Sous-espèces & $\begin{array}{c}\text { No } \\
\text { d'échantillon }\end{array}$ & $\begin{array}{c}\text { No } \\
\text { de couleur } \\
\text { au melloscope } \\
\text { universel }\end{array}$ & $\begin{array}{l}\text { Couleur } \\
\text { du miel }\end{array}$ & $\begin{array}{l}\text { Pourcentage } \\
\text { de trèfle violet }\end{array}$ \\
\hline $\begin{array}{l}\text { A. m. mellifica } \\
\text { (Abeille noire) }\end{array}$ & $\begin{array}{l}1445 \\
1447\end{array}$ & $\begin{array}{r}10 \\
8\end{array}$ & foncée & $\begin{array}{l}0 \\
0\end{array}$ \\
\hline A. m. caucasica & $\begin{array}{l}1441 \\
1446\end{array}$ & $\begin{array}{l}6 \\
6\end{array}$ & moyenne & $\begin{array}{l}31 \\
28\end{array}$ \\
\hline $\begin{array}{c}\text { caucasica } \times \text { ligust } . \\
\text { (hybride) }\end{array}$ & $14 \dot{4} 8$ & 4 & claire & 56 \\
\hline
\end{tabular}


Il est apparu, par la suite, que cette différence dans l'origine florale des miels était grossièrement décelable d'après leur couleur. En effet, si l'on se refère au melloscope universel, on observe une progression inverse de la couleur par rapport au pourcentage de Trè̀le violet. Cette progression est mise en évidence dansle tableau 5

L, a progression de la couleur correspond donc bien à la fois aux différentes sousespèces et aux types de fleurs visités d'une façon préférentielle par chacune d'elles.

\title{
CONCLUSIONS
}

Les résultats de l'analyse pollinique semblent donc confirmer ceux de l'analyse morphologique quantitative. L'analyse pollinique apparaît comme une méthode permettant de confirmer, dans certains cas, une subspéciation. Mais il ne s'agit là que d'une simple remarque car les principaux points confirmés ou mis en évidence sont les suivants :

$\left.\mathrm{I}^{\mathrm{o}}\right)$ Dans les conditions de l'expérience, l'abeille du Jura ne peut pas tirer parti de la sécrétion nectarifère de $T r$. pratense. Toutefois, bien que cela soit fort probable, il n'est pas prouvé que le phénomène se reproduise d'une façon exactement semblable sur l'ensemble du territoire français.

$2^{\circ}$ ) Le problème du butinage sur $T r$. pratense peut être résolu par 1'importation d'A. m. caucasica qui, grâce à la longueur exceptionnelle de son proboscis, peut atteindre facilement les nectaires. Des expériences complémentaires seraient nécessaires avec $A$. $m$. ligustica et des hybrides variées. Des résultats positifs sont très probables.

Enfin, il serait intéressant de tenter une mise en parallèle approfondie de la récolte du pollen et du nectar chez les sous-espèces importées. Toutefois, dans le cas présent, les réactions de l'abeille ne dépendent que de l'anatomie de la fleur; la réponse est nette et le problème est différent. On ne peut le rapprocher, en effet, de l'étude des facteurs de comportements multiples et complexes qui conditionnent la prise de possession des terrains de butinage et le "choix " qualitatif et quantitatif propre à chaque colonie.

Reçu pour publication en février 1963.

\author{
SUMMARY \\ VARIATIONS IN TIE POLLEN ASSOCIATIONS OBSERVED IN HONEYS GATHERED \\ FROM THE SAME AREA BY BEES BELONGING TO DIFFERENT SUB-SPECIES
}

An analysis made of the pollen present in samples of honey taken from colonies of different bee sub-species, but subjected to the same conditions of gathering, confirmed that the Jura region bee (A.m. mellifica) was unable to gather nectar from the Red Clover, but that the sub-species $A . m$. carnica, $A$. m. ligustica and particularly $A . m$. caucasica, as well as a few wild hybrids, possessing a more developed proboscis, were able to gather the nectar secreted by the flowers of Tr. pratense. The classification of the bees into sub-species was made on the basis of a quantitative morphological analysis of five previously defined characteristics. 


\section{RÉFÉRENCES BIBI,IOGRAPHIQUUES}

Dreher K., I950. Beitrage zur Merkmalesuntersuchung der Honigbienne. Z. Bienenforsch., 1 (2), I 7-23. Goetze G., Goetze M., I95I. Rotkleenutzung — Langrüsseligkeit. Die Imme, 10, 25 I-257.

Goetze G., 1956. Methodik der Selektion der Ionibiege auf Langrüsseligkeit. Ins. Soc., 3 (2), $335-346$. Goetze G., 1959-60. Welche Merkmale eignen sich zur Rassenbeurteilung in Korverjaheren. Sudwidelus. scher Imker, 11 (8), 229-230; (9), 268-268; (10), 300-30 I ; 12 (1), I0-I I ; (4) I I I-I I 2 ; (6), 165-I68.

Maurizio A., 1962. Das Pollenbild des Honigs eizelner Völker eines Standes. Disch. Bienewirtschaft, $13(8), 235^{-2} 39$.

Maurizro A., Louveaux J., i 952. Communiqué sur l'activité de la Commission internationale de Botanique apicole de l'U. I.S. B. L'A piculteur sect. scient., fév. 1953, 9-14.

SkovgaARd O.S., 1956. Die kaukasische Honigbiene als Rotkleebestäuber. Tidsskr. Planteavl, 59, 877-887. 